\section{Who should manage primary retinal detachments?}

\author{
M.B. Comer \\ D.K. Newman \\ K.R. Martin \\ A.T. Moore \\ Department of \\ Ophthalmology \\ Addenbrooke's Hospital \\ Cambridge, UK \\ N.D. George \\ Department of \\ Ophthalmology \\ Leeds General Infirmary \\ Leeds, UK
}

\section{B.D.M. Tom}

Centre for Applied Medical

Statistics

Department of Community

Medicine

Institute of Public Health

University of Cambridge, UK

\section{M.B. Comer, MRCP, \\ FRCOphth \\ Department of \\ Ophthalmology \\ Clinic 3 (Box 41) \\ Addenbrooke's Hospital \\ Hills Road \\ Cambridge CB2 2QQ, UK \\ Tel: +44 (0)1223 245151 \\ Fax: $+44(0) 1223217968$}

Funding: None

Proprietary or financial

interests: None

Received: 21 June 1999 Accepted in revised form: 19 January 2000

\section{Abstract}

Purpose To determine whether the outcome of primary retinal reattachment surgery in a subregion is improved by surgery being performed in a specialist vitreoretinal unit (VRU).

Methods A subregional, population-based, retrospective audit cycle of primary retinal reattachment surgery was conducted by independent investigators. The subregion was defined as the catchment area of a teaching hospital (TH) with a specialist VRU and three neighbouring district general hospitals (DGHs). During the initial audit period (January 1989 to December 1990), 142 cases were treated at all four hospitals: TH/VRU (83), DGH-A (15), DGH-B (13), and DGH-C (31). Policy changes after the initial audit led to primary retinal reattachment surgery being predominantly performed by the VRU. During the re-audit period (September 1995 to August 1997), 160 cases were treated at two hospitals: VRU (148) and DGH-C (12). The outcome measure employed was complete retinal reattachment after a single procedure with a minimum follow-up of 12 months.

Results The success rate for primary retinal reattachment surgery in the subregion improved from $76.1 \%$ to $88.8 \%(p=0.006)$ following the policy changes. The success rate of the vitreoretinal specialists in the VRU $(90 \%)$ was greater than the general ophthalmologists in the DGHs (ranging from $47 \%$ to $77 \%$ ), despite case selection by the general ophthalmologists. The number of cases treated by the VRU increased by $156 \%$ in the 6.5 year interval between the two audits due to a widespread change in the model of care for primary retinal detachments (both within and outside the subregion). During the re-audit period, the VRU treated 348 primary retinal detachments (including referrals from outside the subregion), achieving a success rate of $86.8 \%$ with a single procedure and $\mathbf{9 7 . 4} \%$ with further surgery. This primary success rate included 35 cases $(10 \%)$ treated by vitrectomy with silicone oil tamponade who did not undergo silicone oil removal.

Conclusions The outcome of primary retinal
MARIE B. COMER, DOUGLAS K. NEWMAN, NICHOLAS D. GEORGE, KEITH R. MARTIN, BRIAN D.M. TOM, ANTHONY T. MOORE reattachment surgery can be improved if surgery is performed by a specialist VRU. It is suggested that the current standard for retinal reattachment with a single procedure should be set in the region of $85 \%$ to $90 \%$. Changing the model of care so that primary retinal reattachment surgery is predominantly performed by a specialist VRU has important resource implications.

Key words Health care surveys, Population, Retinal detachment, Scleral buckling, Treatment outcome, Vitrectomy

In 1904, the International Congress of Ophthalmology in Lucerne formed the consensus that retinal detachment was untreatable. ${ }^{1}$ This could be considered to have been the first 'standard' of retinal reattachment surgery. The pioneering work of Jules Gonin subsequently demonstrated that retinal detachments could be treated with a success rate of around $60 \%{ }^{2}$ The success rate has since improved with advances in surgical technique including binocular indirect ophthalmoscopy, ${ }^{3}$ scleral buckling, ${ }^{4}$ intraocular tamponade, ${ }^{5,6}$ and pars plana vitrectomy. ${ }^{7}$ Most contemporary reports of large consecutive case series indicate that a success rate of greater than $90 \%$ is now achievable, though $10-20 \%$ of cases require more than one operation for retinal reattachment. ${ }^{8}$

The primary retinal reattachment rate is of great importance in the assessment of retinal reattachment surgery. Retinal reattachment with a single procedure is generally associated with a better visual outcome and reduced patient morbidity. ${ }^{8}$ However, the success rate of primary retinal reattachment surgery has only been assessed by a few studies (Table 1). While these reports have mostly emanated from dedicated vitreoretinal units (VRUs), a significant proportion of primary retinal reattachment surgery in the United Kingdom is performed by general ophthalmologists. Little information is available on the outcome of retinal reattachment surgery performed in nonspecialist centres. The current trend towards sub-specialisation in ophthalmology is leading to retinal reattachment surgery being 
Table 1. Outcome of primary retinal reattachment surgery: published consecutive case series

\begin{tabular}{|c|c|c|c|c|c|c|}
\hline Location of VRU & $\begin{array}{l}\text { Time } \\
\text { period }\end{array}$ & $\begin{array}{l}\text { No. of } \\
\text { cases }\end{array}$ & Surgical techniques & $\begin{array}{l}\text { Primary } \\
\text { success rate }\end{array}$ & $\begin{array}{l}\text { Final } \\
\text { success rate }\end{array}$ & Reference \\
\hline UK (London) & 1967-72 & 452 & Scleral buckle & $75 \%$ & $88 \%$ & Chignell et al. ${ }^{19}$ \\
\hline USA (Iowa City) & 1969-73 & 863 & Scleral buckle & $76 \%$ & $89 \%$ & Rachal and Burton ${ }^{10}$ \\
\hline USA (San Francisco) & 1967-79 & 1008 & Scleral buckle & $84 \%$ & $92 \%$ & Grizzard et al. ${ }^{11}{ }^{11}$ Hilton et al. ${ }^{12}$ \\
\hline Sweden (Örebro) & $1971-81$ & 590 & Scleral buckle & $65 \%$ & $78 \%$ & Törnquist and Törnquist ${ }^{13}$ \\
\hline Finland (Helsinki) & $1978-81$ & 352 & $\begin{array}{l}\text { Scleral buckle }(99 \%) \\
\text { Vitrectomy }(1 \%)\end{array}$ & $\mathrm{N} / \mathrm{R}$ & $87 \%^{d}$ & Laatikainen et al. ${ }^{14}$ \\
\hline USA (Oklahoma City) & $(1984)^{a}$ & 662 & Scleral buckle ${ }^{b}$ & $91 \%$ & $94 \%$ & Wilkinson and Bradford ${ }^{15}$ \\
\hline India (Madras) & $1986-89$ & 601 & Scleral buckle ${ }^{c}$ & $86 \%$ & $90 \%$ & Sharma et al. ${ }^{16}$ \\
\hline UK (London) & $(1997)^{a}$ & 153 & $\begin{array}{l}\text { Scleral buckle }(66 \%) \\
\text { Vitrectomy }(34 \%)\end{array}$ & $80 \%$ & $97 \%$ & Sullivan et al. ${ }^{17}$ \\
\hline
\end{tabular}

These studies report referral-based case series treated in dedicated VRUs (except for one study ${ }^{13}$ which reports a population-based survey of primary retinal reattachment surgery). N/R, not reported.

${ }^{a}$ Date of publication is given because the time period for the study was not specified.

${ }^{b}$ Cases requiring vitrectomy were excluded (indications for vitrectomy and number treated by vitrectomy not specified).

${ }^{c}$ Cases requiring vitrectomy were excluded, which comprised giant retinal tear, severe proliferative vitreoretinopathy and severe ocular trauma (number of cases treated by vitrectomy not specified).

${ }^{d}$ Final success rate of $87 \%$ was only achieved in the time period $1980-81$.

increasingly performed by VRUs. The aim of this study was to investigate whether this results in an improved success rate for retinal reattachment surgery.

\section{Subjects and methods}

A subregional, population-based, retrospective audit cycle of the outcome of primary retinal reattachment surgery was conducted by independent investigators. The subregion was defined as the catchment area of a teaching hospital (TH) with a specialist VRU and three neighbouring district general hospitals (DGHs). The catchment population of the hospitals was: TH (280 000), DGH-A (210 000), DGH-B (140 000) and DGH-C (230 000). The VRU also received subregional, regional and national vitreoretinal referrals. The inclusion criterion for both audit periods was any patient residing in the subregion who underwent surgery for a primary rhegmatogenous retinal detachment (excluding retinal detachment secondary to penetrating ocular trauma). Case ascertainment was achieved in each hospital by reviewing the theatre log book and identifying any patient who had undergone a vitreoretinal procedure during each audit period. The medical records of these patients were then reviewed in order to identify those patients who had undergone primary retinal reattachment surgery. The retrieval rate for medical records was $95 \%$ for the initial audit and $90 \%$ for the re-audit.

The initial audit, which was performed in 1993, assessed the 24 month period January 1989 to December $1990 .^{18}$ A total of 142 primary retinal detachments (140 patients) was treated at four hospitals in the subregion during this period: TH/VRU (83), DGH-A (15), DGH-B (13) and DGH-C (31). Primary retinal reattachment surgery at the $\mathrm{TH}$ was mainly performed under the care of one vitreoretinal specialist in the VRU, but 5 patients $(6 \%)$ were treated under the care of other consultant ophthalmologists. At the DGHs, primary retinal reattachment surgery was performed under the care of one consultant ophthalmologist at DGH-A, one consultant ophthalmologist at DGH-B and two consultant ophthalmologists at DGH-C. The DGHs also selectively referred some primary retinal detachments to the VRU during the initial audit period: DGH-A (9), DGH-B (3) and DGH-C (4).

The re-audit assessed the 24 month period September 1995 to August 1997. A total of 160 primary retinal detachments (156 patients) was treated at two hospitals in the subregion during this period: VRU (148) and DGH-C (12). The findings of the initial audit had led to several policy changes in the subregion. Firstly, DGH-A and DGH-B stopped performing retinal reattachment surgery and referred all cases to the VRU. Secondly, all retinal reattachment surgery at the $\mathrm{TH}$ was now performed by the VRU under the care of two vitreoretinal specialists. Thirdly, only one consultant ophthalmologist at DGH-C now performed retinal reattachment surgery. DGH-C also selectively referred 8 primary retinal detachments to the VRU during the reaudit period.

The effect of these policy changes was determined by analysing the outcome of primary retinal reattachment surgery in the subregion during both audit periods. The outcome measure employed was complete retinal reattachment after a single procedure with a minimum follow-up of 12 months. For cases treated by primary vitrectomy with silicone oil tamponade, this outcome measure was applied after silicone oil removal (if performed). Visual acuity was not selected as an outcome measure because it is affected by multiple confounding factors other than the surgical procedure itself. These include the nature of the retinal detachment (macular involvement and duration of detachment), postoperative complications (such as cataract and macular pucker) and coexistent ocular morbidity.

In addition, the current success rate of all primary retinal reattachment surgery performed by the VRU was determined. A total of 348 primary retinal detachments was treated by the VRU during the re-audit period 
Table 2. Characteristics of retinal detachments in the initial audit period

\begin{tabular}{|c|c|c|c|c|}
\hline \multirow[b]{2}{*}{ Characteristic } & \multicolumn{4}{|c|}{ No. $(\%)$ of retinal detachments } \\
\hline & $\begin{array}{l}\text { DGH-A } \\
(n=15)\end{array}$ & $\begin{array}{c}\text { DGH-B } \\
(n=13)\end{array}$ & $\begin{array}{l}\text { DGH-C } \\
(n=31)\end{array}$ & $\begin{array}{c}\text { TH/VRU } \\
(n=83)\end{array}$ \\
\hline \multicolumn{5}{|l|}{ Type of retinal break } \\
\hline Horseshoe tear ${ }^{a}$ & $10(67)$ & $10(77)$ & $23(74)$ & $51(61)$ \\
\hline Round hole & $3(20)$ & $2(15)$ & $8(26)$ & $18(22)$ \\
\hline Dialysis & 1 (7) & 0 & 0 & $6 \quad(7)$ \\
\hline Retinoschisis & 0 & 0 & 0 & $1(1)$ \\
\hline None found & $1 \quad(7)$ & $1(8)$ & $0 \quad(0)$ & $7(8)$ \\
\hline Macula off & $12(80)$ & $12(92)$ & $25(81)$ & $53(64)$ \\
\hline Total retinal detachment & $1(7)$ & 0 & $4(13)$ & $7(8)$ \\
\hline Aphakia & $2(13)$ & 0 & $1(3)$ & $6(7)$ \\
\hline Pseudophakia & $3(20)$ & $6(46)$ & $4(13)$ & $11(13)$ \\
\hline
\end{tabular}

Details regarding the presence of proliferative vitreoretinopathy were not available for the initial audit period.

${ }^{a}$ Includes retinal detachments due to horseshoe tears in combination with either round holes or macular holes.

(September 1995 to August 1997), comprising 148 cases from within the subregion and 200 cases referred from outside the subregion. The success rate of each surgical technique was assessed (scleral buckling, vitrectomy with gas tamponade and vitrectomy with silicone oil tamponade). The outcome of primary retinal reattachment surgery performed by trainee ophthalmologists was also analysed to determine the effect of supervision by a vitreoretinal specialist.

The chi-square test with Yates' continuity correction was used to compare nominal data. Fisher's exact test was used if the chi-square test was not valid because of small expected cell counts. Multifactorial analysis was performed using multiple logistic regression with backward elimination.

\section{Results}

The characteristics of the retinal detachments treated at each hospital during both audit periods are given in Tables 2 and 3. These comprise recognised pre-operative risk factors for failure at primary retinal reattachment

Table 3. Characteristics of retinal detachments in the re-audit period

\begin{tabular}{|c|c|c|}
\hline \multirow[b]{2}{*}{ Characteristics } & \multicolumn{2}{|c|}{ No. (\%) of retinal detachments } \\
\hline & $\begin{array}{l}\text { DGH-C } \\
(n=12) \\
\end{array}$ & $\begin{array}{c}\text { VRU } \\
(n=148)\end{array}$ \\
\hline \multicolumn{3}{|l|}{ Type of retinal break } \\
\hline Horseshoe tear ${ }^{a}$ & $9(75)$ & $112(76)$ \\
\hline Round hole & $1(8)$ & $20(14)$ \\
\hline Dialysis & $1(8)$ & $9(6)$ \\
\hline Retinoschisis & 0 & $4 \quad(3)$ \\
\hline Primary macular hole & 0 & $1(1)$ \\
\hline None found & $1(8)$ & $2(1)$ \\
\hline PVR (grade C1 or worse) ${ }^{19}$ & 0 & $6(4)$ \\
\hline Macula off & $8(67)$ & $107(72)$ \\
\hline Total retinal detachment & $1(8)$ & $8(5)$ \\
\hline Aphakia & 0 & $3(2)$ \\
\hline Pseudophakia & $5(42)$ & $15(10)$ \\
\hline
\end{tabular}

PVR, proliferative vitreoretinopathy.

${ }^{a}$ Includes retinal detachments due to horseshoe tears in combination with either round holes or macular holes. surgery. ${ }^{8}$ The retinal detachments treated at each hospital did not differ significantly regarding these characteristics during either audit period.

\section{Outcome of primary retinal reattachment surgery in the subregion: completion of audit cycle}

The outcome of primary retinal reattachment surgery during each audit period is given in Table 4. During the initial audit period there was a significant difference between the success rates of the four hospitals $(p=0.01)$. The TH/VRU had a better success rate than the DGHs collectively ( $84 \%$ vs $64 \% ; p=0.01$ ). This finding led to several policy changes in the subregion, resulting in primary retinal reattachment surgery only being performed by the VRU and DGH-C during the re-audit period. Following the policy changes, there was a significant improvement in the success rate of primary retinal reattachment surgery in the subregion: $76.1 \%$ $(108 / 142)$ during the initial audit period versus $88.8 \%$ $(142 / 160)$ during the re-audit period $(p=0.006)$. The VRU continued to have better success rate than DGH-C during the re-audit period, but this did not reach statistical significance $(p=0.27)$. However, the re-audit had a power of only $30 \%$ for detecting a difference at the $5 \%$ significance level because of the small number of cases treated at DGH-C.

Table 4. Outcome of primary retinal reattachment surgery in each audit period

\begin{tabular}{lcc}
\hline & \multicolumn{2}{c}{ Retinal reattachment with a single procedure } \\
\cline { 2 - 3 } Hospital & Initial audit period & Re-audit period \\
\hline DGH-A & $47 \%(7 / 15)$ & N $/ A$ \\
DGH-B & $77 \%(10 / 13)$ & N/A \\
DGH-C & $68 \%(21 / 31)$ & $75 \%(9 / 12)$ \\
TH/VRU $^{a}$ & $84 \%(70 / 83)$ & $90 \%(133 / 148)$ \\
\hline
\end{tabular}

N/A, not applicable.

${ }^{a}$ During the initial audit period, primary retinal reattachment surgery at the TH was performed by the VRU for 78 patients and under the care of other consultant ophthalmologists for 5 patients. During the re-audit period, all primary retinal reattachment surgery at the TH was performed by the VRU. 


\begin{tabular}{lcccc}
\hline & \multicolumn{2}{c}{ Initial audit period } & & \multicolumn{2}{c}{ Re-audit period } \\
\cline { 2 - 4 } Reason for failure & $\begin{array}{c}\text { All DGHs } \\
(n=21)\end{array}$ & $\begin{array}{c}\text { TH/VRU } \\
(n=13)\end{array}$ & $\begin{array}{c}\text { DGH-C } \\
(n=3)\end{array}$ & 2 \\
New/missed retinal break & 8 & 7 & $15)$ \\
Inadequate scleral buckle & 6 & - & 1 \\
Complications of SRF drainage & 3 & - & - \\
No retinal break found & 2 & 4 & - \\
Proliferative vitreoretinopathy & 1 & - & - \\
Macular hole & 1 & - & - \\
Recurrence of retinoschisis cavity & - & 1 & -
\end{tabular}

SRF, subretinal fluid.

The techniques used by the VRU for primary retinal reattachment surgery changed in the interval between the audit periods. Vitrectomy procedures accounted for only $8 \%(7 / 83)$ of cases during the initial audit period compared with $46 \%(68 / 148)$ of cases during the re-audit period. The DGHs only used scleral buckling procedures during both audit periods. However, the success rate of primary retinal reattachment surgery at the TH/VRU was not significantly different between the two audit periods $(84 \%$ vs $90 \% ; p=0.31)$. This suggests that the principal factor accounting for the improved success rate of primary retinal reattachment surgery in the subregion was the change in centres performing surgery rather than any change in surgical technique at the VRU.

Primary vitrectomy with silicone oil tamponade was used by the VRU to treat 7 cases during the initial audit period and 27 cases during the re-audit period. During the initial audit period, 5 cases treated by primary vitrectomy with silicone oil tamponade were successful but none underwent silicone oil removal because of poor potential for visual improvement. During the re-audit period, 24 cases treated by primary vitrectomy with silicone oil tamponade were successful. Silicone oil was removed in 9 of these cases resulting in one redetachment. The reasons for retaining silicone oil in the remaining 15 successful cases were: poor potential for visual improvement (10), macular hole (2), patient preference (2) and significant risk of re-detachment (1).

Trainee ophthalmologists performed primary retinal reattachment surgery during both audit periods. The number of cases performed by trainees during the initial audit period was: DGH-A (1), DGH-B (nil), DGH-C (1) and TH/VRU (32). The grade of trainee at the TH/VRU was senior registrar (19), registrar (12) and senior house officer (1). A vitreoretinal specialist supervised only 3 of these cases. The number of cases performed by trainees during the re-audit period was: DGH-C (nil) and VRU (51). The grade of trainee at the VRU was fellow (36), specialist registrar (12) and senior house officer (3). A vitreoretinal specialist supervised 14 of these cases.

The reasons for failure of primary retinal reattachment surgery are given in Table 5 . New or missed retinal breaks were the most common reasons for failure in all hospitals during both audit periods. During the initial audit period, the DGHs performed further surgery on 13 cases and the VRU performed further surgery on 18 cases (including 7 failed cases referred from the DGHs). During the re-audit period, DGH-C performed further surgery on 2 cases and the VRU performed further surgery on 16 cases (including 1 failed case referred from the DGH-C).

The final anatomical success rate for the subregion was $96 \%(137 / 142)$ in the initial audit and 99\% (158/160) in the re-audit $(p=0.26)$. There was no significant difference in the final anatomical success rate for cases initially treated by the DGHs compared with cases initially treated by the VRU. During the initial audit period, the final anatomical success rate was $95 \%(56 / 59)$ for cases initially treated by the DGHs and $98 \%(81 / 83)$ for cases initially treated by the TH/VRU $(p=0.65)$. During the re-audit period, the final anatomical success rate was $92 \%(11 / 12)$ for cases initially treated by DGH-C and $99 \%(147 / 148)$ for cases initially treated by the VRU $(p=0.15)$.

\section{Resource implications of performing primary retinal reattachment surgery at the vitreoretinal unit}

The VRU also received vitreoretinal referrals from DGHs outside the subregion. In many of these DGHs there was a policy change in the 6.5 year interval between the two audits, resulting in more primary retinal detachments being referred to the VRU rather than such surgery being performed locally with subsequent referral of any failed cases. This widespread change in practice led to a considerable increase in primary retinal reattachment surgery performed at the VRU: 136 cases during the initial audit period ( 83 within the subregion and 53 outside the subregion) compared with 348 cases during the re-audit period (148 within the subregion and 200 outside the subregion). This increase in workload required the appointment of a second vitreoretinal specialist.

\section{Current success rate for all primary retinal reattachment surgery performed by the vitreoretinal unit}

A total of 348 primary retinal detachments was treated by the VRU during the re-audit period (148 within the subregion and 200 outside the subregion). The success rate for all primary retinal reattachment surgery performed by the VRU was $86.8 \%(302 / 348)$. The preoperative characteristics of these retinal detachments are given in Table 6. Multiple logistic regression analysis 
Table 6. Characteristics of all primary retinal detachments treated by the specialist VRU during the re-audit period (148 cases within the subregion and 200 cases outside the subregion)

\begin{tabular}{lrr}
\hline Characteristic & No. (\%) of retinal detachments \\
\hline Type of retinal break $^{\prime}$ & 266 & $(76)$ \\
Horseshoe tear & \\
Round hole & 50 & $(14)$ \\
Dialysis & 12 & $(3)$ \\
Retinoschisis & 6 & $(2)$ \\
Giant retinal tear & 5 & $(1)$ \\
Primary macular hole & 3 & $(1)$ \\
None found & 6 & $(2)$ \\
PVR (grade C1 or worse) & 13 & $(4)$ \\
Macula off & 237 & $(68)$ \\
Total retinal detachment & 22 & $(6)$ \\
Aphakia & 6 & $(2)$ \\
Pseudophakia & 35 & $(10)$ \\
\hline
\end{tabular}

PVR, proliferative vitreoretinopathy.

${ }^{a}$ Includes retinal detachments due to horseshoe tears in combination with either round holes or macular holes.

demonstrated that macular involvement was the only pre-operative characteristic associated with an increased risk of failure of primary retinal reattachment surgery ( $p=0.008 ;$ OR $=3.7 ; 95 \%$ CI 1.4 to 9.8 ).

The success rate of each technique used for primary retinal reattachment surgery was: scleral buckling $88 \%$ $(158 / 179)$, vitrectomy with gas tamponade $87 \%(83 / 95)$ and vitrectomy with silicone oil tamponade $82 \%(61 / 74)$ $(p=0.45)$. Silicone oil was removed from 28 cases treated by primary vitrectomy with silicone oil tamponade at a mean of 7 months post-operatively, resulting in 2 re-detachments. The reasons for retaining silicone oil in the remaining 35 successful cases were: poor potential for visual improvement (24), patient preference (6), macular hole (3), anaesthetic risk (1) and significant risk of redetachment (1).

Trainee ophthalmologists performed 33\% (115/348) of primary retinal reattachment surgery at the VRU. The grade of trainee was fellow (82), specialist registrar (27) and senior house officer (6). The success rate for surgery performed by the vitreoretinal specialists was: scleral buckling procedure $87 \%(101 / 116)$, vitrectomy with gas tamponade $90 \%(54 / 60)$ and vitrectomy with silicone oil tamponade $86 \%$ (49/57). The success rate for surgery performed by trainees was: scleral buckling procedure $90 \%$ (57/63), vitrectomy with gas tamponade $83 \%$ (29/35) and vitrectomy with silicone oil tamponade $71 \%$ $(12 / 17)$. The success rate for surgery performed by trainees was $100 \%(32 / 32)$ with supervision compared with $80 \%(66 / 83)$ without supervision $(p=0.01)$. Trainees were therefore able to achieve good results in a VRU provided surgery was directly supervised by a vitreoretinal specalist.

Further surgery was performed on 38 cases that failed primary retinal reattachment surgery. Retinal reattachment was achieved with one further procedure for 33 cases and two further procedures for 4 cases. The final anatomical success rate was therefore $97.4 \%$
$(339 / 348)$. Of the remaining 9 cases, 7 achieved macular reattachment with a stable inferior retinal detachment.

\section{Discussion}

This study was a population-based retrospective audit cycle of the outcome of primary retinal reattachment surgery. Retinal detachments occurring in a defined subregion were treated by four hospitals, comprising a TH with a specialist VRU and three DGHs. The vitreoretinal specialists in the VRU achieved a success rate of $90 \%$ with a single procedure. In contrast, the general ophthalmologists in the DGHs had lower success rates, ranging from $47 \%$ to $77 \%$. These results were achieved despite the general ophthalmologists treating selected retinal detachments (between $11 \%$ and $40 \%$ of cases presenting to the DGHs were referred to the VRU). The audit cycle demonstrated that the success rate for primary retinal reattachment surgery in the subregion was improved by a change in practice resulting in surgery being predominantly performed by the VRU (76.1\% vs $88.8 \%, p=0.006)$. While this audit suffers the inherent limitations of a retrospective study, it does enable a more reliable assessment of clinical practice than a prospective audit. Prospective audit can induce changes in normal clinical practice because clinicians are aware that an audit is in progress ('The Hawthorne Effect' $\left.^{\prime}\right){ }^{20,21}$

Until recently, the standard model of care for primary retinal detachments was for 'simple' cases to be treated by general ophthalmologists and 'complicated' cases to be referred to vitreoretinal specialists. ${ }^{22}$ This distinction was based on the predicted response of the retinal detachment to a scleral buckling procedure, with vitrectomy procedures being indicated for 'complicated' cases. For this model of care to achieve satisfactory results, general ophthalmologists must be able to identify 'simple' cases accurately from pre-operative assessment. However, events can occur during surgery that convert a 'simple' case into a 'complicated' case. For example, severe scleromalacia or additional retinal breaks may be unexpectedly found that render a scleral buckling procedure inappropriate. Any ophthalmologist performing retinal reattachment surgery should therefore have the necessary facilities and expertise to alter the surgical approach if required.

This distinction between 'simple' and 'complicated' retinal detachments is now becoming less relevant because of the increasing use of vitrectomy techniques to treat primary retinal detachments. ${ }^{23}$ Current indications for vitrectomy techniques include advanced proliferative vitreoretinopathy, ${ }^{24}$ retinal breaks that are technically difficult to treat with scleral buckles (giant retinal tears, macular breaks, large or posterior horseshoe tears, and complex arrangements of retinal breaks) $)^{25-38}$ and poor retinal visualisation due to media opacities. ${ }^{27-29}$ The benefits of vitrectomy compared with scleral buckling procedures continue to be explored. ${ }^{30}$ The use of vitrectomy procedures increased considerably in this 
study during the 6.5 year interval between the two audits (from $8 \%$ to $46 \%$ of operations). An accompanying improvement in the success rate for primary retinal reattachment surgery at the VRU was observed $(84 \%$ vs $90 \%$ ), though this did not reach statistical significance. Another recent study of primary retinal reattachment surgery also reported that $34 \%$ of cases are currently treated by vitrectomy. ${ }^{17}$

The VRU in this study treated a significant proportion of primary retinal detachments by vitrectomy with silicone oil tamponade. The ocular indications for silicone oil tamponade were: advanced proliferative vitreoretinopathy; giant retinal tear; primary macular hole; retinoschisis; and some horseshoe tears. In particular, the VRU employed the technique of using silicone oil as a surgical instrument (rather than simply as post-operative tamponade) in the management of certain retinal detachments. ${ }^{31}$ Psychosocial factors also dictated the use of silicone oil rather than gas for intraocular tamponade in some cases. These included patients with retinal detachment in an only eye (to allow rapid visual rehabilitation), patients wishing to fly shortly after surgery, and patients whose poor general health rendered surgery under general anaesthetic a significant risk.

There are important resource implications if the local model of care for primary retinal detachments is changed so that more cases are treated by a VRU. The VRU in this study received subregional, regional and national vitreoretinal referrals. In addition to policy changes within the subregion, many of the referring DGHs outside the subregion also changed their policy and referred increasing numbers of primary retinal detachments to the VRU during the interval between the two audits. Consequently, the number of primary retinal detachments treated by the VRU increased by $156 \%$ over a 6.5 year interval. The increase in primary retinal reattachment surgery greatly exceeded any decrease in repeat surgery for failed cases and required the appointment of a second vitreoretinal specialist. Adequate resource allocation must therefore be provided if a local model of care for primary retinal detachments is changed so that the increase in referrals to the VRU can be accommodated.

This study also investigated the issue of training in vitreoretinal surgery. The success rate for primary retinal reattachment surgery performed by trainees was considerably better when surgery was performed under supervision by a vitreoretinal specialist $(100 \%$ with supervision vs $80 \%$ without supervision; $p=0.01$ ). The benefit of supervision is probably greater than suggested by these figures due to differences in case-mix and grade of trainee. As a result of this finding, the VRU has changed its policy so that all retinal reattachment surgery by trainees is performed under direct supervision by a vitreoretinal specialist. It is considered that trainees can achieve experience in vitreoretinal surgery without unfavourable results provided there is both appropriate case selection and direct supervision by a vitreoretinal specialist.
Primary retinal reattachment surgery still fails to achieve the goal of a $100 \%$ success rate. The commonest cause of failure in this study was new or missed retinal breaks, but these could not be reliably distinguished. While missed retinal breaks should always be regarded as an avoidable cause of failure, new retinal breaks are more problematic. Studies are required to determine whether new retinal breaks occur in a predictable fashion, raising the possibility of preventive action. Proliferative vitreoretinopathy continues to be reported as a common cause of failure. ${ }^{8}$ In recent years, the prognosis of failed cases with proliferative vitreoretinopathy has improved considerably though the visual outcome is usually limited. ${ }^{24}$ During the re-audit period of this study, however, the incidence of failure due to proliferative vitreoretinopathy was only $0.3 \%$ (1/348 cases). This finding suggests that proliferative vitreoretinopathy can be virtually eliminated by appropriate primary retinal reattachment surgery.

All ophthalmologists performing retinal reattachment surgery should regularly audit their results to ensure a satisfactory standard is being achieved. No nationally agreed standard for primary retinal reattachment surgery currently exists, but this is likely to change with the introduction of clinical governance. There are only a few published reports of the outcome of primary retinal reattachment surgery that have mostly emanated from dedicated VRUs (Table 1). The reported success rates range from $65 \%$ to $91 \%$, though such figures are influenced by case-mix and publication bias. It has been suggested that a primary retinal reattachment rate of $75 \%$ is a reasonable standard. ${ }^{17}$ However, the VRU in this population-based survey achieved a $90 \%$ success rate for primary retinal reattachment surgery. Similar primary retinal reattachment rates have been reported by two other VRUs in the United Kingdom: $88 \%$ in the Mersey region $^{32}$ and $85 \%$ in the Newcastle region. ${ }^{33}$ These findings suggest that the current standard for primary retinal reattachment surgery should be set in the region of $85 \%$ to $90 \%$. This standard may be best achieved by specialist VRUs.

We are grateful to all the consultant ophthalmologists in the subregion for their help and co-operation in this study. We also wish to acknowledge A.B. Callear, A. Halkias and A. Ruigrok who assisted in the data collection for the initial audit period.

\section{References}

1. Duke-Elder S, Dobree JH. Detachment and folding of the retina. In: Duke-Elder S, editor. System of ophthalmology, vol.10. Diseases of the retina. St Louis: Mosby-Year Book, 1967:771.

2. Gonin J. Le décollement de la rétine. Lausanne: Payot et Cie, 1934.

3. Schepens CL. A new ophthalmoscope demonstration. Trans Am Acad Ophthalmol Otolaryngol 1947;51:298.

4. Custodis E. Die Behandlung der Netzhautablösung durch umschriebene Diathermiekoagulation und einer mittels Plombenaufnähung erzeugten Eindellung der Sklera im Bereich des Risses. Klin Monatsbl Augenheilkd 1956;129:476. 
5. Cibis PA, Becker B, Okun E, Canaan S. The use of liquid silicone in retinal reattachment surgery. Arch Ophthalmol 1962;68:590-9.

6. Norton EW. Intraocular gas in the management of selected retinal detachments. Trans Am Acad Ophthalmol Otolaryngol 1973;77:85-98.

7. Machemer R, Buettner H, Norton EW, Parel JM. Vitrectomy: a pars plana approach. Trans Am Acad Ophthalmol Otolaryngol 1971;75:813.

8. Wilkinson CP, Rice TA. Michels retinal detachment, 2nd ed. St Louis: Mosby, 1997.

9. Chignell AH, Fison LG, Davies EW, Hartley RE, Gundry MF. Failure in retinal detachment surgery. Br J Ophthalmol 1973;57:525-30.

10. Rachal WF, Burton TC. Changing concepts of failures after retinal detachment surgery. Arch Ophthalmol 1979;97:480-3.

11. Grizzard WS, Hilton GF, Hammer ME, Taren D. A multivariate analysis of anatomic success of retinal detachments treated with scleral buckling. Graefes Arch Clin Exp Ophthalmol 1994;232:1-7.

12. Hilton GF, McLean EB, Brinton DA. Retinal detachment: principles and practice, 2nd ed. San Francisco: American Academy of Ophthalmology, 1995.

13. Törnquist $R$, Törnquist P. Retinal detachment: a study of a population-based patient material in Sweden 1971-1981. III. Surgical results. Acta Ophthalmol 1988;66:630-6.

14. Laatikainen L, Harju H, Tolppanen EM. Post-operative outcome in rhegmatogenous retinal detachment. Acta Ophthalmol 1985;63:647-55.

15. Wilkinson CP, Bradford RH. Complications of draining subretinal fluid. Retina 1984;4:1-4.

16. Sharma T, Challa JK, Ravishankar KV, Murugesan R. Scleral buckling for retinal detachment: predictors for anatomic failure. Retina 1994;14:338-43.

17. Sullivan PM, Luff AJ, Aylward GW. Results of primary retinal reattachment surgery: a prospective audit. Eye 1997;11:869-71.

18. George ND, Callear AB, Halkias A, Ruigrok A, Moore AT. Should all primary retinal detachments be referred to a specialist vitreoretinal unit for surgery? Presented at the Royal College of Ophthalmologists Annual Congress, 1994.

19. The Retina Society Terminology Committee. The classification of retinal detachment with proliferative vitreoretinopathy. Ophthalmology 1983;90:121-5.
20. Irvine D, Irvine S, editors. How to do it: data and methods 1 . In: Making sense of audit. Oxford: Radcliffe Medical Press, 1991:55.

21. Roethlisberger FJ, Dickson WJ. In: Management and the worker. An account of a research program conducted by the Western Electric Company, Hawthorne Works, Chicago. Cambridge, MA: Harvard University Press, 1943:526-8.

22. Leaver PK. Retinal detachments: when to refer. Identifying the complicated case. Eye 1989;3:754-8.

23. Leaver P. Expanding the role of vitrectomy in retinal reattachment surgery. Br J Ophthalmol 1993;77:197.

24. The Silicone Study Group. Vitrectomy with silicone oil or perfluoropropane gas in eyes with severe proliferative vitreoretinopathy: results of a randomised clinical trial. Silicone Study Report 2. Arch Ophthalmol 1992;110:780-92.

25. Billington BM, Leaver PK. Vitrectomy and fluid/silicone oil exchange for giant retinal tears: results at 18 months. Graefes Arch Clin Exp Ophthalmol 1986;224:7-10.

26. Gonvers M, Machemer R. A new approach to treating retinal detachments with macular hole. Am J Ophthalmol 1982;94:468-72.

27. Gartry DS, Chignell AH, Franks WA, Wong D. Pars plana vitrectomy for the treatment of rhegmatogenous retinal detachment uncomplicated by advanced proliferative vitreoretinopathy. Br J Ophthalmol 1993;77:199-203.

28. Hakin KN, Lavin MJ, Leaver PK. Primary vitrectomy for rhegmatogenous retinal detachment. Graefes Arch Clin Exp Ophthalmol 1993;231:344-6.

29. Wong D, Billington BM, Chignell AH. Pars plana vitrectomy for retinal detachment with unseen retinal holes. Graefes Arch Clin Exp Ophthalmol 1987;225:269-71.

30. Bartz-Schmidt KU, Kirchhof B, Heimann K. Primary vitrectomy for pseudophakic retinal detachment. Br J Ophthalmol 1996;80:346-9.

31. Scott JD. Silicone oil as an instrument. In: Ryan SJ, editor. Retina, vol 3. St Louis: CV Mosby, 1994:2181-9.

32. Wong D, McGalliard J. Are we getting better at treating retinal detachment? Technology, referral pattern or primary care? Eye 1997;11:763-70.

33. Ramsay A, Cottrell DG. Triple cycle audit of retinal detachment surgery. Presented at the Royal College of Ophthalmologists Audit Meeting, 1998. 